\title{
Improving the conductivity of hole injection layer by heating PEDOT:PSS
}

\author{
Kao-Hua Tsai ${ }^{1}$, Shu-Chia Shiu ${ }^{1}$ and Ching-Fuh $\operatorname{Lin}^{1,2,3}$ \\ ${ }^{1}$ Institute of Photonics and Optoelectronics, National Taiwan University, ${ }^{2}$ Graduate Institute of \\ Electronics Engineering, National Taiwan University, and ${ }^{3}$ Department of Electrical Engineering, \\ National Taiwan University \\ No.1, Sec. 4, Roosevelt Road, Taipei, 106, Taiwan, R.O.C. \\ Phone: 02-23635251 ext405, Fax: 02-23677467, E-mail: J96921009@ntu.edu.tw \\ Graduate Institute of Photonics and Optoelectronics, Graduate Institute of Electronics Engineering \\ and Department of Electrical Engineering, National Taiwan University, Taipei, Taiwan, 10617 \\ R.O.C. \\ E-mail address: cflin@cc.ee.ntu.edu.tw
}

(NSC-96-2221-E-002-277-MY3, NSC-96-2218-E-002-025)

\begin{abstract}
Poly(3,4-ethylenedioxythiophene): poly(4-styrenesulfonic acid) (PEDOT:PSS) is a common material of hole injection layer used in polymer light emitting diodes (PLEDs) and organic solar cells. It can improve the efficiency of the charge collection at the anode. It has been reported that adding glycerol to PEDOT:PSS could increase the conductivity and improve the efficiency of PLEDs and organic solar cells. However, it is less noticed that the conductivity could be improved when the solution of PEDOT was heated before deposition. Here we experimented different concentrations of glycerol into PEDOT:PSS to make G-PEDOT:PSS solution, and heated the G-PEDOT:PSS solution at different temperatures before deposition. The solutions are then spin-coated on the glass and annealed at $140{ }^{\circ} \mathrm{C}$. The conductivity was then measured and compared. The experiments showed that the conductivity of pure PEDOT:PSS slightly increased for 2-3 times, while the G-PEDOT:PSS increased over two orders of magnitudes. The conductivity increased with the heating temperature before deposition. The enhancement of the conductivity of the G-PEDOT:PSS film was higher than that of the pure PEDOT:PSS film. The overall conductivity increase for over three orders of magnitude. The reason is because the high temperature causes the glycerol and PEDOT:PSS to mix evenly. This is helpful for the swelling and aggregation of colloidal PEDOT-rich particles, forming a highly conductive network. When G-PEDOT:PSS resistance is reduced, it may not only increase the hole collection ability, but also replace ITO as the anode layer due to its advantages of low production cost and high work function.
\end{abstract}

Keywords: PEDOT:PSS, glycerol, PLED

\section{INTRODUCTION}

Conductive polymeric materials have highly attracted attention over the past several years mainly due to their potential advantage of low-cost, large-area, light-weight and vacuum-free fabrication [1,2]. Conductive polymeric materials not only replace lots of metal and inorganic conducting materials but also become the critical key in the modern industry and advanced technology.

PEDOT:PSS or Poly(3,4-ethylenedioxythiophene) poly(styrenesulfonate) is used as a conducting polymer. This compound is generally applied as a dispersion of gelled particles in water. A conductive layer on glass is obtained by spreading a layer of the dispersion on the surface usually by spin coating and driving out the water by heat [3]. The advantage of PEDOT:PSS layer is virtually transparent, colorless, high conductivity $\left(10^{-6}\right.$ to $\left.10^{1} \mathrm{~S}-\mathrm{cm}-1\right)$, good environment stability, thin film of easy process , preventing electrostatic discharges during film rewinding, and reducing dust buildup on the negatives after processing. It is an ideal material with applications that include polymer light-

Organic Photovoltaics IX, edited by Zakya H. Kafafi, Paul A. Lane,

Proc. of SPIE Vol. 7052, 70521B, (2008) $\cdot 0277-786$ X/08/\$18 $\cdot$ doi: $10.1117 / 12.795039$

Proc. of SPIE Vol. 7052 70521B-1 
emitting diodes (PLEDS), thin-film transistors, electrochromic devices [4,5], super-capacitors [6], actuators [7], biosensors. [8], and photo diodes.

Indium tin oxide (ITO) is the material of choice for a high-work function transparent conducting electrical contact in the majority of cases. But ITO has disadvantage of inequalities in the surface. It is usually covered by a thin buffer layer of the oxidatively doped, cationic conductive polymer PEDOT:PSS in order to smooth the ITO surface, to decrease the hole barrier, the leakage current, and to increase current injection.

However, the conductivity of commonly used PEDOT:PSS (Baytron P) is five orders of magnitude lower than ITO. When the electric hole passes to the PEDOT:PSS level, the power conversion efficiency (PCE) decreases if the resistance of PEDOT:PSS is high [ 9 ]. Decreasing the resistance of PEDOT:PSS has attracted considerable attention due to the performance for Organic photovoltaic devices (PVs).

The conductivity of PEDOT:PSS can increase by decreasing the concentration of PSS in the PEDOT:PSS solution [10]. Decreasing the concentration of PSS would make the quality of PEDOT:PSS film worse and damage the performance of device. Recently, processing PEDOT:PSS with polyalcohols or other inert secondary dopants leads to an enhancement of conductivity of up to 2 orders of magnitudes. High conductivity can decrease the series resistance (Rs) and increase the short current of devices [11], but it has a disadvantage of decreasing work function of PEDOT:PSS [12] and it increases leakage current with increasing polyalcohol. Besides, the quality of PEDOT:PSS film would be worse.

In this paper, we fixed the amount of glycerol which was doped into PEDOT:PSS, and then heated the solution which had PEDOT:PSS and glycerol. We found this way can make the conductivity of doped PEDOT:PSS have a large elevation . The doped PEDOT:PSS after heating leads to an enhancement of conductivity over three orders of magnitude, and its film quality was better than solution without heating.

\section{EXPERIMENT}

First, four sets of solution were fabricated. For solution set 1(S1), the PEDOT:PSS (Baytron P) is pristine without glycerol. For solution set 2(S2), 30mg glycerol was doped in $1 \mathrm{ml}$ PEDOT:PSS . For solution set 3(S3), 60mg glycerol was doped in $1 \mathrm{ml}$ PEDOT:PSS . For solution set 4(S4), 90mg glycerol was doped in $1 \mathrm{ml}$ PEDOT:PSS. We stirred the solutions with different doping concentrations of glycerol in the PEDOT:PSS about one hour and deposited the solution on glasses by spin coating at $4000 \mathrm{rpm}$ and dried at $140{ }^{\circ} \mathrm{C}$ for $10 \mathrm{~min}$.

Subsequently, we take the pristine PEDOT:PSS, the PEDOT:PSS doped 30mg/c.c. glycerol, and the PEDOT:PSS doped $60 \mathrm{mg} /$ c.c. glycerol divided into five, respectively. We set the solutions from five to nineteen (S5-S19). S5, S6, S7, S8, S9 are pristine. PEDOT:PSS. S10, S11, S12, S13, S14 are PEDOT:PSS doped 30mg/c.c. glycerol. S15, S16, S17, S18, S19 are PEDOT:PSS doped $60 \mathrm{mg} /$ c.c. glycerol. After stirring the solutions for one hour, we sealed the solution and heated the solutions with different temperature for $30 \mathrm{~min} . \mathrm{S} 5, \mathrm{~S} 10, \mathrm{~S} 15$ was not heated $\left(\mathrm{R} . \mathrm{T} .=20^{\circ} \mathrm{C}\right.$ ). S6, S11, S16 were heated at $40^{\circ} \mathrm{C}$. S7, S12 and S17 were heated at $80{ }^{\circ} \mathrm{C}$. S8, S13 and S18 were heated at $120{ }^{\circ} \mathrm{C}$. S9, S14 and S19 were heated at $160^{\circ} \mathrm{C}$. Then we deposited the solution on glasses by spin coating at $4000 \mathrm{rpm}$ and dried at $140{ }^{\circ} \mathrm{C}$ for $10 \mathrm{~min}$.

The resistance of PEDOT:PSS was measured by four-point probe station and a Keithley 2400 source-measure unit. The thickness of the film was measured by $\alpha$-step. Besides, we measured the transmissions of S5 to S9 and S15 to S19. Finally, we compared the difference of transmissions with different heating temperatures and different doping concentrations of glycerol in the PEDOT:PSS.

\section{RESULT}

The resistivities and thicknesses of sets 1 to 4 are presented in Table1. The sheet resistances are $2.4 * 106 \Omega / \square$, $1.6 * 104 \Omega / \square, 8.3 * 103 \Omega / \square, 5.9 * 103 \Omega / \square$, the thicknesses are $90 \mathrm{~nm}, 80 \mathrm{~nm}, 75 \mathrm{~nm}, 72 \mathrm{~nm}$, and the resistivities are $21.6 \Omega$ $\mathrm{cm}, 0.128 \Omega-\mathrm{cm}, 0.062 \Omega-\mathrm{cm}, 0.0425 \Omega-\mathrm{cm}$, respectively. It shows that the conductivity increased and the thickness decreased with the increasing concentration of glycerol in PEDOT:PSS. In figure1, we plotted the resistivity against the concentration of glycerol in the PEDOT:PSS. It shows that few glycerol doped in PEDOT:PSS can increase many conductivities. 
The resistivities and thicknesses of sets 5 to 19 are presented in Table2. The sheet resistances of sets 5 to 9 heated at different temperatures are $2.4 * 10^{6} \Omega / \square, 1.83^{*} 10^{6} \Omega / \square, 1.62 * 10^{6} \Omega / \square, 1.42^{*} 10^{6} \Omega / \square, 1.26^{*} 10^{6} \Omega / \square$, respectively. The thickness is $90 \mathrm{~nm}, 91 \mathrm{~nm}, 95 \mathrm{~nm}, 98 \mathrm{~nm}, 100 \mathrm{~nm}$, respectively, and the resistivity is $21.6 \Omega-\mathrm{cm}, 16.8 \Omega-\mathrm{cm}, 15.4 \Omega-\mathrm{cm}$, $13.8 \Omega-\mathrm{cm}, 12.6 \Omega-\mathrm{cm}$, respectively. The results of sets 5 to 9 show that the conductivity slightly increases and the thickness slightly decreases with the heating temperatures of solutions.

The sheet resistances of sets 10 to 14 heated at different temperatures are $1.6^{*} 10^{4} \Omega / \square, 1.1^{*} 10^{3} \Omega / \square, 8.1^{*} 10^{3} \Omega / \square$, $5.75^{*} 10^{3} \Omega / \square, 4.25^{*} 10^{3} \Omega / \square$, respectively, for all thickness of about $80 \mathrm{~nm}$, which corresponds to resistivities of $0.128 \Omega$ $\mathrm{cm}, 0.085 \Omega-\mathrm{cm}, 0.064 \Omega-\mathrm{cm}, 0.046 \Omega-\mathrm{cm}, 0.034 \Omega-\mathrm{cm}$, respectively.

The sheet resistances of sets 15 to 19 heated at different temperatures are $8.3^{*} 10^{3} \Omega / \square, 4.5^{*} 10^{3} \Omega / \square, 3^{*} 10^{3} \Omega / \square$, $2.3^{*} 10^{3} \Omega / \square, 1.8^{*} 10^{3} \Omega / \square$, respectively, for all thickness of about $75 \mathrm{~nm}$, which corresponds to resistivities of $0.062 \Omega$-cm, $0.034 \Omega-\mathrm{cm}, 0.022 \Omega-\mathrm{cm}, 0.017 \Omega-\mathrm{cm}, 0.014 \Omega-\mathrm{cm}$, respectively. The results of sets 10 to 19 show that the conductivity increases with the heating temperatures of solutions more than the pristine PEDOT:PSS. Besides, thicknesses of these samples do not differ from each other.

We plotted the resistivity against the heating temperatures with different G-PEDOT in figure 3, 4 and 5 As shown in the figures, the resistivities decrease fast when the solution heating at $40{ }^{\circ} \mathrm{C}$ and $80{ }^{\circ} \mathrm{C}$, then it reduced slowly with increasing temperatures.

The resulting transmittance spectra on glass, together with the heating temperatures are shown in figure 5 and 6 . Note that the transmittance decreases at high heating temperature, and it obviously reduces at $40^{\circ} \mathrm{C}$ to $80^{\circ} \mathrm{C}$ and $80^{\circ} \mathrm{C}$ to 120 ${ }^{\circ} \mathrm{C}$. Figures 5 and 6 also show that the transmittance at long wavelengths $(800 \mathrm{~nm}-1100 \mathrm{~nm})$ reduced more than at short wavelengths (300 nm-600 nm ). Besides, the transmittance of doped PEDOT:PSS was higher than of pristine PEDOT:PSS by heating temperature.

Table 1. The characteristic of PEDOT:PSS which was doped with different concentrations of glycerol.

\begin{tabular}{|c|c|c|c|c|}
\hline sample & $\begin{array}{c}\text { glycerol/PEDOT } \\
(\mathrm{mg} / \text { c.c. })\end{array}$ & $\begin{array}{c}\text { sheet resistances } \\
(\Omega / \square)\end{array}$ & $\begin{array}{c}\text { thickness } \\
(\mathrm{nm})\end{array}$ & $\begin{array}{c}\text { resistivity } \\
(\Omega \text {-cm })\end{array}$ \\
\hline $\mathrm{S} 1$ & 0 & $2.4 * 10^{6}$ & 90 & 21.6 \\
\hline $\mathrm{S} 2$ & 30 & $1.6 * 10^{4}$ & 80 & 0.128 \\
\hline $\mathrm{S} 3$ & 60 & $8.3 * 10^{3}$ & 75 & 0.062 \\
\hline $\mathrm{S} 4$ & 90 & $5.9^{*} 10^{3}$ & 72 & 0.043 \\
\hline
\end{tabular}




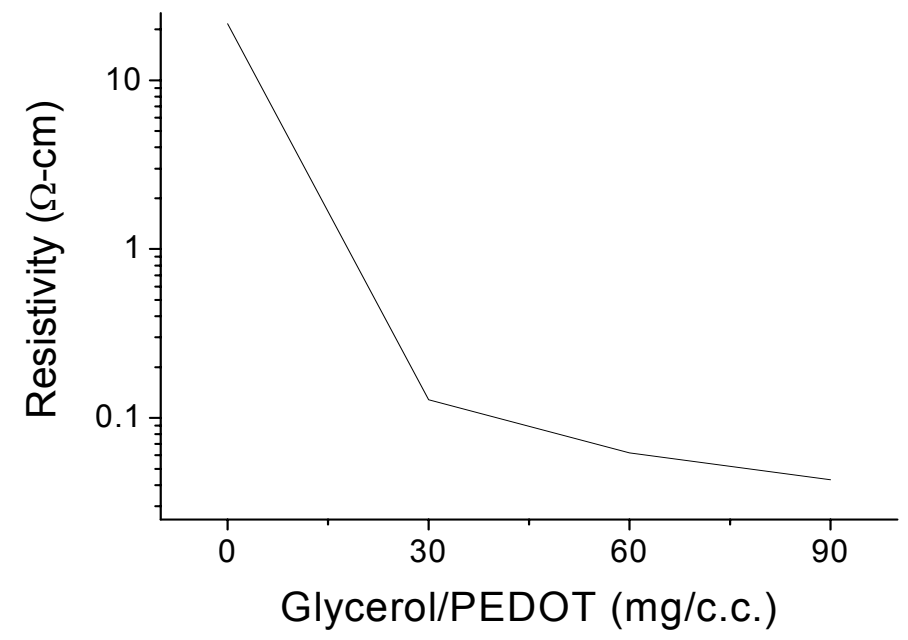

Fig. 1. This figure is the resistivity of solutions with different doping concentrations of glycerol in the PEDOT:PSS .

Table 2. The characteristic of layers which were made of pristine PEDOT:PSS or different concentrations G-PEDOT .

\begin{tabular}{|c|c|c|c|c|c|}
\hline sample & $\begin{array}{l}\text { glycerol/PEDOT } \\
\text { (mg/c.c.) }\end{array}$ & $\begin{array}{l}\text { temperature } \\
\left({ }^{\circ} \mathrm{C}\right)\end{array}$ & $\begin{array}{l}\text { sheet resistances } \\
\qquad(\Omega / \square)\end{array}$ & $\begin{array}{l}\text { thickness } \\
\text { (nm) }\end{array}$ & $\begin{array}{l}\text { resistivity } \\
\text { ( } \Omega-\mathrm{cm})\end{array}$ \\
\hline S5 & 0 & RT & $2.4^{*} 10^{6}$ & 90 & 21.6 \\
\hline S6 & 0 & 40 & $1.83 * 10^{6}$ & 91 & 16.8 \\
\hline S7 & 0 & 80 & $1.62 * 10^{6}$ & 95 & 15.4 \\
\hline S8 & 0 & 120 & $1.42 * 10^{6}$ & 98 & 13.8 \\
\hline S9 & 0 & 160 & $1.26^{*} \times 10^{6}$ & 100 & 12.6 \\
\hline S10 & 30 & RT & $1.6^{*} 10^{4}$ & 80 & 0.128 \\
\hline S11 & 30 & 40 & $1.1 * 10^{4}$ & 80 & 0.085 \\
\hline S12 & 30 & 80 & $8.1 * 10^{3}$ & 80 & 0.064 \\
\hline S13 & 30 & 120 & $5.75^{*} 10^{3}$ & 80 & 0.046 \\
\hline S14 & 30 & 160 & $4.25^{*} 10^{3}$ & 80 & 0.034 \\
\hline S15 & 60 & RT & $8.3 * 10^{3}$ & 75 & 0.062 \\
\hline S16 & 60 & 40 & $4.5^{*} 10^{3}$ & 75 & 0.034 \\
\hline S17 & 60 & 80 & $3 * 10^{3}$ & 75 & 0.022 \\
\hline S18 & 60 & 120 & $2.3 * 10^{3}$ & 75 & 0.017 \\
\hline S19 & 60 & 160 & $1.8 * 10^{3}$ & 75 & 0.014 \\
\hline
\end{tabular}




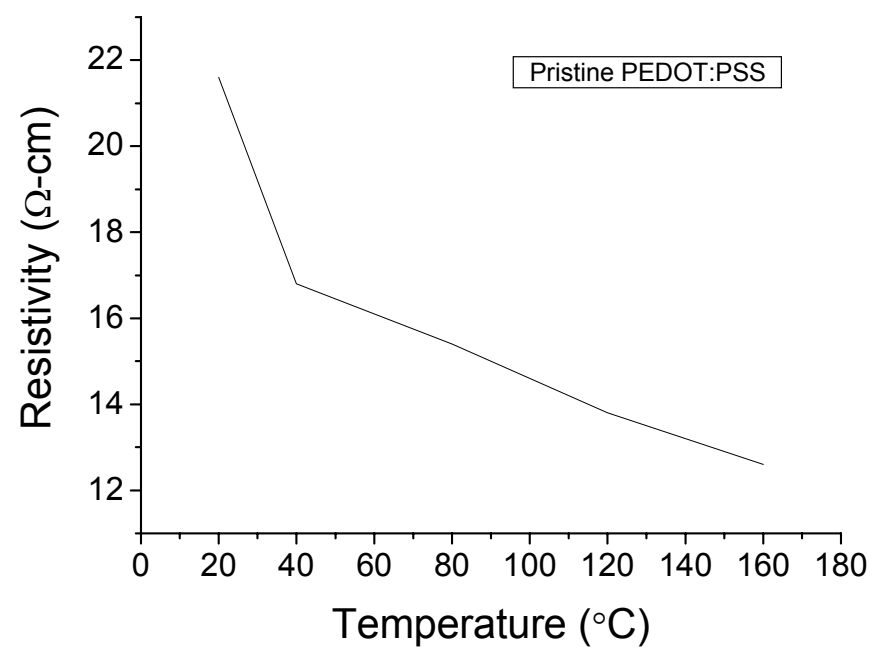

Fig. 2. This figure is the resistivity of pristine PEDOY:PSS heated at different temperature.

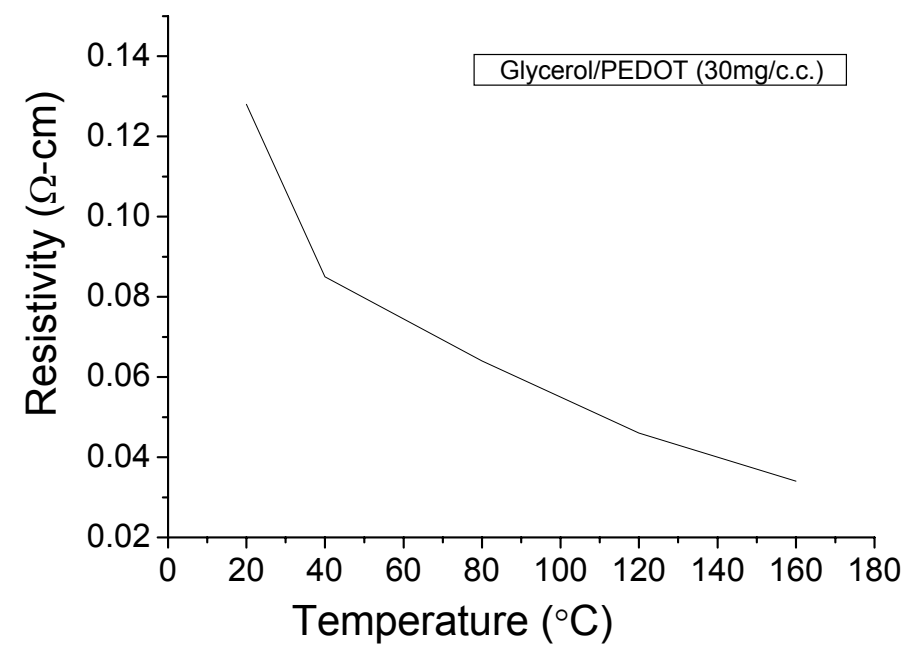

Fig. 3. This figure is the resistivity of PEDOT with $30 \mathrm{mg} / \mathrm{c} . c$. glycerol heated at different temperature. 


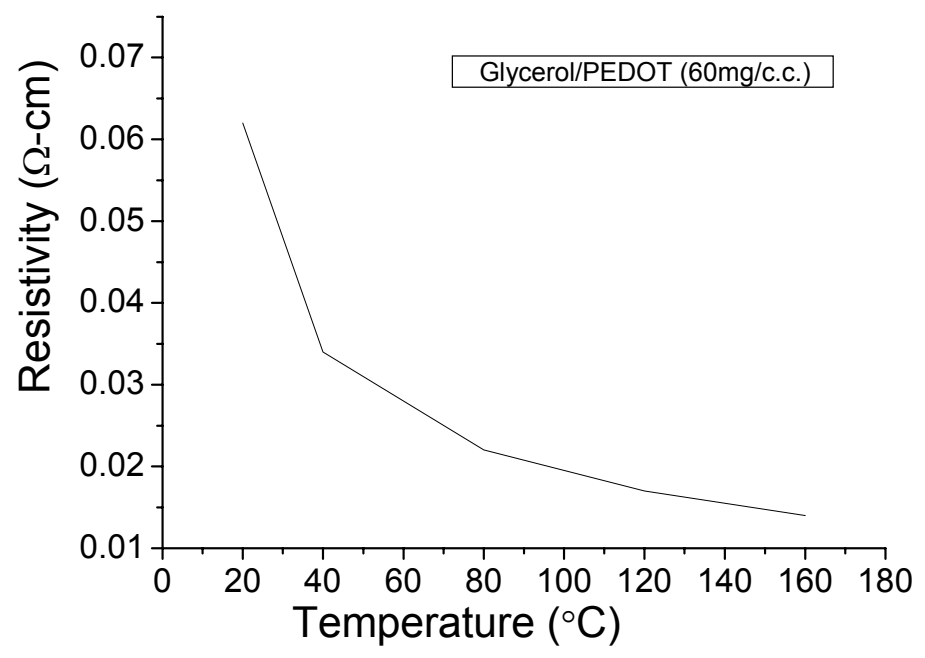

Fig. 4. This figure is the resistivity of PEDOT with $60 \mathrm{mg} / \mathrm{c} . c$. glycerol heated at different temperature.

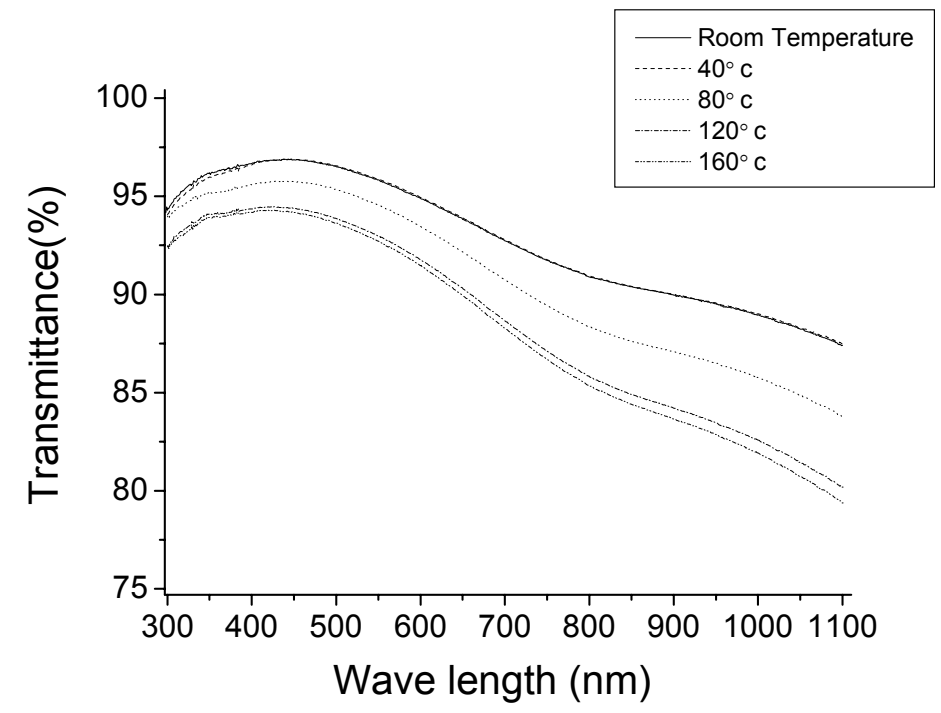

Fig. 5. This figure is the transmittance of sets 5 to 9 . Note that the transmittance decreased at high heating temperature, and it obviously decreased at $40{ }^{\circ} \mathrm{C}$ to $80{ }^{\circ} \mathrm{C}$ and $80{ }^{\circ} \mathrm{C}$ to $120^{\circ} \mathrm{C}$. The transmittances decreased $4-6 \%$ at long wavelengths $(800 \mathrm{~nm}-1100 \mathrm{~nm})$ while decreased $2-4 \%$ at short wavelengths $(300 \mathrm{~nm}-600 \mathrm{~nm})$. 


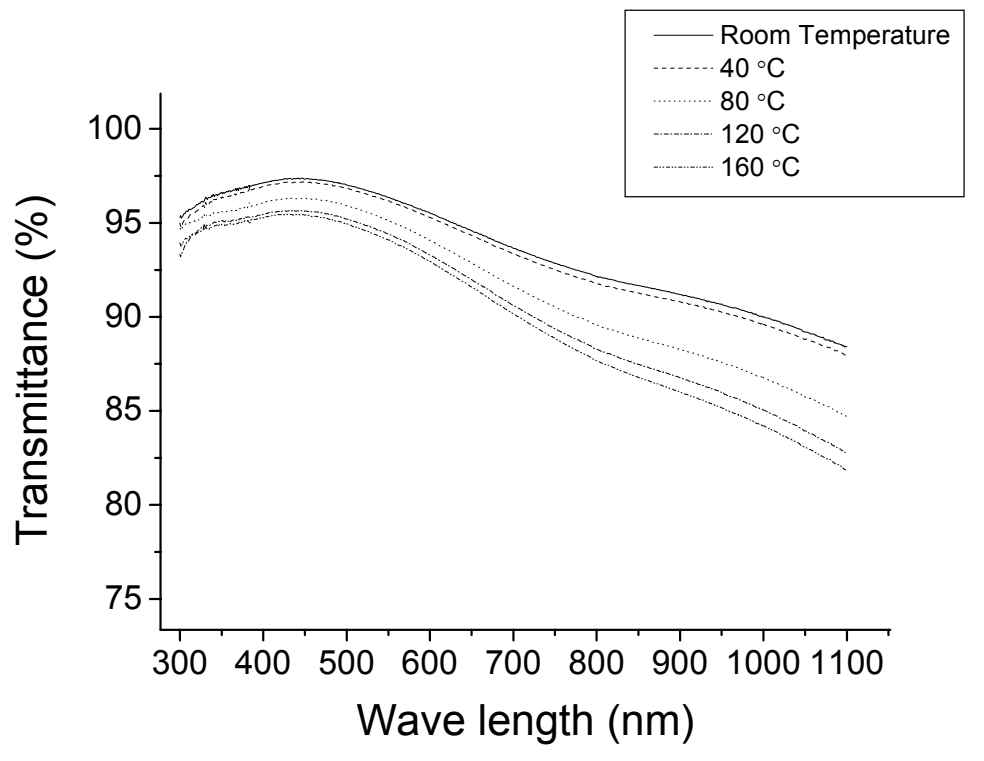

Fig. 6. This figure is the transmittance of sets 15 to 19 . Note that the transmittance decreased at high heating temperature, and it obviously decreased at $40{ }^{\circ} \mathrm{C}$ to $80{ }^{\circ} \mathrm{C}$ and $80{ }^{\circ} \mathrm{C}$ to $120^{\circ} \mathrm{C}$. The transmittances decreased $3-5 \%$ at long wavelengths $(800 \mathrm{~nm}-1100 \mathrm{~nm})$ while decreased 1-3\% at short wavelengths $(300 \mathrm{~nm}-600 \mathrm{~nm})$. The average transmittance Tavg is larger than sets 5 to 9 .

\section{DISCUSSION}

The resistivity of the pristine PEDOT:PSS is twice more than that of the heated PEDOT:PSS. The resistivity of PEDOT:PSS doped $90 \mathrm{mg} /$ c.c. glycerol is 500 times higher than that of the pristine PEDOT:PSS. Heating the PEDOT:PSS solution doped glycerol can reduce the resistivity five times. Doping and heating the solution makes the conductivity 2000 times higher than pristine PEDOT:PSS.

The improved conductivity of doped PEDOT:PSS is due to swelling and aggregation of the colloidal PEDOT-rich particles, forming a highly conductive network [10]. The reason of improved conductivity by heating PEDOT:PSS is because the high temperature causes the glycerol and PEDOT:PSS to mix evenly. This is helpful for the enhanced swelling and aggregation of colloidal PEDOT-rich particles, forming a higher conductive network than pristine PEDOT:PSS.

Heating the solution can increase the conductivity of the PEDOT:PSS film, but it decreases some transmittance. With heating treatment, the transmittance of doped PEDOT:PSS is less than that of pristine PEDOT:PSS. Although the high temperature reduced transmittances, the average transmittance $T_{\text {avg }}$ for the limited absorption range of most materials between 300 and $800 \mathrm{~nm}$ could still be sufficient.

\section{CONCLUSION}

In comclusion, we demonstrate that heating doped PEDOT:PSS solution can reduce the resistivity to $1.4 * 10^{-2} \Omega$-cm. Its resistivity is 70 times higher than ITO, whose resistivity is $2^{*} 10^{-4} \Omega-\mathrm{cm}$. When the G-PEDOT:PSS resistance is reduced, 
it may not only increase the hole collection ability, but also replace ITO as the anode layer due to its advantages of low production cost and high work function.

\section{ACKNOWLEDGEMENTS}

This project was supported by the National Science Council, Taiwan, Republic of China, with Grant No.: NSC962221-E-002-277-MY3, and NSC96-2218-E-002-025.

\section{REFERENCES}

[1] M. Granstrom, M. Berggren, D. Pede, O. Inganas, M. R. Andersson, T. Hjertberg, O. Wennerstrom, Supramol. Sci. (1997), 4, 27.

[2] J.Bharathan, Y. Yang, Appl. Phys. Lett. (1998), 72, 2660.

[3] http://en.wikipedia.org/wiki/PEDOT:PSS

[4] L. B. Groenendaal, G. Zotti, P. H. Aubert, S. M. Waybright, J. R. Reynolds, Adv. Mater. 2003, 15, 855.

[5] S. Kirchmeyer, K. Reuter, J. Mater. Chem. (2005), 15.

[6] B. E. Conway, Electrochemical Supercapacitor, Scientific Fundamentals and Technological Applications, Kluwer Academic/Plenum Press, New York 1999.

[7] E. W. H. Jager, E. Smela, O. Inganäs, Science 2000, 290, 1540.

[8] M. Gerard, A. Chaubey, B. D. Malhotra, Biosens. Bioelectron. 2002, 17, 345.

[9] Chu-Jung Ko, Yi-Kai Lin, Fang-Chung Chen, and Chi-Wei Chu, Appl. Phys. Lett. 90, 063509 (2007)

[10] H. J. Snaith, H. Kenrick, M. Chiesa, R. H. Friend, Polymer (2005), 46, 2573.

[11] F. Zhang, M. Johansson, M.R. Andersson, J.C. Hummelen, O. Inganas, Adv. Mater. 14 (2002) 662.

[12] S.-H. Jin et al. / Solar Energy Materials \& Solar Cells 91 (2007) 1187-1193 